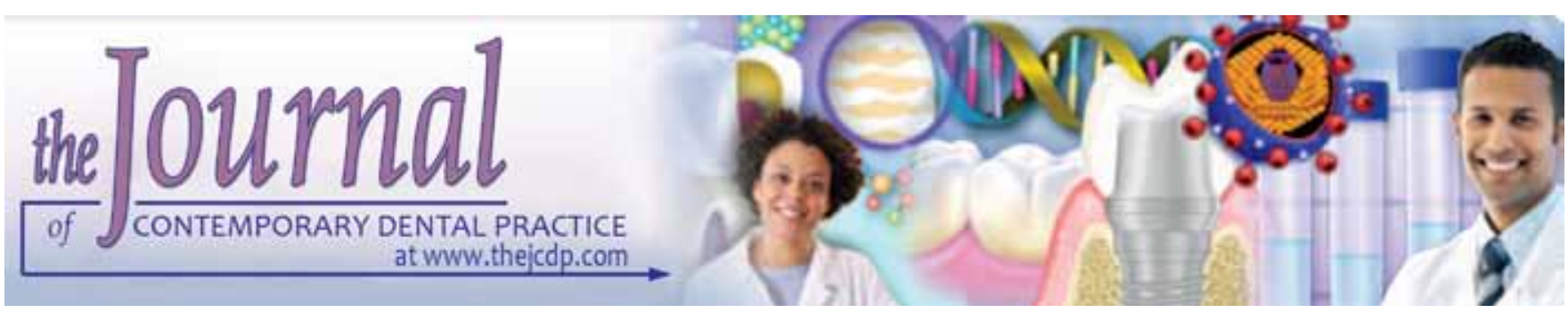

\title{
Erosion Potential of Tooth Whitening Regimens as Evaluated with Polarized Light Microscopy
}

\author{
${ }^{1}$ Patrick Brambert, ${ }^{2}$ Fang Qian, ${ }^{3}$ So Ran Kwon
}

\begin{abstract}
Aims: Tooth whitening is a widely utilized esthetic treatment in dentistry. With increased access to over-the-counter (OTC) systems concerns have been raised as to potential adverse effects associated with overuse of whitening materials. Therefore, this study aimed to evaluate enamel erosion due to different whitening regimens when used in excess of recommended guidelines.
\end{abstract}

Materials and methods: Extracted human teeth $(n=66)$ were randomly divided into 11 groups ( $n=6 /$ group). Specimens were exposed to OTC products: Crest Whitestrips and 5-minute natural white and a do-it-yourself (DIY) strawberry whitening recipe. Within each regimen, groups were further divided per exposure time: specimens receiving the recommended product dosage; 5 times the recommended dosage; and 10 times the recommended dosage. Negative and positive controls were treated with grade 3 water and $1.0 \%$ citric acid, respectively. Specimens were nail-varnished to limit application to a $1 \times 4 \mathrm{~mm}$ window. Following treatment, specimens were sectioned and erosion (drop in $\mu \mathrm{m}$ ) measured using polarized light microscopy. Two-sample t-test was used to detect difference in amount of enamel erosion between negative and positive groups, while one-way analysis of variance (ANOVA), followed by post hoc Dunnett's test was used to detect difference between set of treatment groups and negative control groups or among all experimental groups.

Results: There was significant difference in mean amount of enamel erosion $(p<0.0001)$. Mean enamel erosion for positive

\footnotetext{
${ }^{1}$ Department of Dentistry, University of lowa College of Dentistry Lowa City, United States

${ }^{2}$ Department of Preventive and Community Dentistry, Division of Biostatistics and Research Design, University of lowa College of Dentistry, Lowa City, United States

${ }^{3}$ Department of Center for Dental Research, Loma Linda University School of Dentistry, Loma Linda, California, United States

Corresponding Author: So Ran Kwon, Associate Professor Center for Dental Research, Loma Linda University, School of Dentistry, 11175 Campus, Street Suite A 1010, Loma Linda 92350, California United States, Phone: 909558 8069, e-mail: sorankwon@llu.edu
}

control group was significantly greater than that for negative control group (23.50 vs $2.65 \mu \mathrm{m})$. There was significant effect for type of treatments on enamel erosion $[F(9,50)=25.19 ; p$ $<0.0001]$. There was no significant difference between the negative control and each of treatment groups ( $p>0.05$ for all instances), except for Natural White_10 times treatment group $(p<0.0001)$ that was significantly greater than the negative control group (14.82 vs $2.65 \mu \mathrm{m})$.

Conclusion: Caution is advised when using certain over-thecounter products beyond recommended guidelines as there is potential for enamel erosion.

Clinical significance: Enamel erosion due to the overuse of whitening products varies for different modalities and products. Therefore, caution is advised when using certain over-thecounter products beyond recommended guidelines, as there is potential for enamel erosion.

Keywords: Abuse, Erosion, Polarized light microscopy, Tooth whitening.

How to cite this article: Brambert P, Qian F, Kwon SR. Erosion Potential of Tooth Whitening Regimens as Evaluated with Polarized Light Microscopy. J Contemp Dent Pract 2015;16(11):921-925.

\section{Source of support: Nil}

\section{Conflict of interest: None}

\section{INTRODUCTION}

Tooth whitening has become one of the most utilized esthetic procedures in dentistry over the past 20 years. ${ }^{1}$ Due to its popularity, various modalities of whitening teeth have become available treatment choices to the public. This includes do-it-yourself (DIY) whitening, over-the-counter (OTC) products, professionally dispensed patient applied peroxide based materials, and professionally applied light accelerated whitening products. $^{2}$

Various types of tooth whiteners have been found to be effective in improving tooth color when the manufacturer's given protocol is followed. ${ }^{3}$ While overnight 
trays with conventional gels have been found to be the most effective in retaining a lighter tooth shade, overthe-counter products could still produce a comparable result when used for a longer time period. ${ }^{3}$ According to Auschill et al, the use of customized trays with peroxide based material required about seven applications to observe a six shade tab improvement in tooth color using the Vita Classic shade guide (VITA Zahnfabrik, Bad Sackingen, Germany). Comparatively, OTC whitening strips required about 32 applications to produce a similar whitening outcome. ${ }^{4}$ Therefore, evidence of efficacy of different whitening modalities seems to be well established.

With the increased demand and use of OTC products that are not supervised by dental heath professionals special emphasis has been laid on investigating the effects of OTC whitening products on enamel erosion. One recent study has shown that OTC products that are used following manufacturer guidelines do not increase the surface roughness of enamel. ${ }^{5,6}$ In fact, it was also found that whitening following manufacturer guidelines did not increase the susceptibility of enamel to erosion or abrasion. ${ }^{7}$ However there are as many studies showing the opposite, i.e. whitening caused a reduction in microhardness, ${ }^{8}$ increased surface roughness, ${ }^{9}$ and accompanied increased susceptibility to localized bacteria colonization on the tooth surface. ${ }^{10}$ It has been pointed out that the contradictory results of various studies may be attributed to the differences in vitro protocols with respect to replicating the dynamic in vivo environment, the study design, type and concentration of peroxide compound, pH and exposure time. ${ }^{11}$

Another important aspect that has not been explored is related to the erosive effects of whitening when products are continuously abused and overused. The risk of patient abuse of whitening agents has increased over time, with adolescents being a particularly at risk group for overuse of whitening agents. ${ }^{12}$ There is only limited research on this topic and therefore no clearly defined threshold values available for dental professionals to determine exactly how much excess whitening leads to compromise, or critical enamel erosion. The International Organization for Standardization (ISO) defines this critical point as three times the amount of erosion seen in an hour of acid attack by citric acid with a $\mathrm{pH}$ of 3.9. ${ }^{13}$ Therefore, the purpose of this study was to exceed the whitening dosages set by manufacturers of various whitening modalities to determine how much excess application of whitening products would lead to critically eroded enamel. The hypothesis to be tested was that use of whitening materials beyond manufacturer's recommendation would not increase erosion amount.

\section{MATERIALS AND METHODS}

Sample selection and preparation: Extracted sound human molar teeth without identifiers $(n=66)$ were collected and stored in $0.1 \%$ Thymol solution at $4^{\circ} \mathrm{C}$. Teeth were cleaned of gross debris and placed in artificial saliva for 24 hours at $37^{\circ} \mathrm{C}$ prior to initiating the experiment. All teeth were painted with nail varnish (Sally Hansen, New York, NY, USA) to expose a standardized $1 \times 4 \mathrm{~mm}$ treatment area.

Tooth whitening protocol by experimental groups: Sixtysix teeth were randomized and equally divided into 11 groups ( $n=6 /$ group) and exposed to the whitening agents as summarized in Table 1. Specimens were exposed to OTC products: Crest 3D Intensive Whitestrips (Procter and Gamble, Cincinnati, OH, USA), 5-minute natural white (Lornamead Inc., Tonawanda, NY, USA), and a DIY strawberry whitening recipe consisting of a puree of strawberry (15 gm) mixed with baking soda (2.5 gm) (Arm and Hammer Baking Soda, Church and Dwight Co., Inc, Princeton, NJ, USA) for 5 minutes followed by a final brush with a soft toothbrush (Colgate Oral Pharmaceuticals, Inc., New York, NY, USA) for 30 seconds. The procedure was repeated two more times at 5-day intervals. Within each regimen, groups were further divided per exposure time: specimens receiving the recommended product dosage; 5 times the recommended dosage; and 10 times the recommended dosage. Negative and positive controls were treated with grade 3 water and $1.0 \%$ citric

Table 1: Tooth whitening protocol by experimental group

\begin{tabular}{lll}
\hline Treatment group & Treatment regimen & Mean drop $\mu m(S D)$ \\
\hline NC: Water of grade 3 & 1 application (60 minutes) at $35^{\circ} \mathrm{C}$ & $2.65(1.11)$ \\
SB_1: Strawberry mixture & 3 applications (5 minutes each) at 5-day intervals & $2.95(1.46)$ \\
SB_5 & 5 times recommended protocol & $3.16(1.48)$ \\
SB_10 & 10 times recommended protocol & $3.10(1.50)$ \\
CWS_1: Crest 3D intensive & 1 daily application $(2$ hours) for 7 days & $3.04(1.53)$ \\
CWS_5 & 5 times recommended protocol & $3.10(1.99)$ \\
CWS_10 & 10 times recommended protocol & $2.80(1.45)$ \\
NW_1: Natural white & 1 daily application (5 min) for 7 days & $2.75(1.11)$ \\
NW_5 & 5 times recommended protocol & $3.62(0.95)$ \\
NW_10 & 10 times recommended protocol & $14.82(3.93)$ \\
PC: Citric acid & 1 application (60 minutes) at 35 ${ }^{\circ} \mathrm{C}$ & $23.50(3.91)$ \\
\hline
\end{tabular}


acid, respectively according to guidelines of ISO 28399. Following treatment, specimens were sectioned and erosion (drop in $\mu \mathrm{m}$ ) was measured using polarized light microscopy.

Polarized light microscopy: Upon treatment teeth were cut vertically with a microtome (ISOMET 1000, Buehler, Lake Bluff, Illinois, USA) in buccolingual orientations to yield sections of $40 \mu \mathrm{m}$ thickness to be observed with polarized light microscopy (Olympus BHT, Tokyo, Japan). A digital scale was used to measure the amount of erosion in each testing condition at three points and averaged.

Data analysis: A two-sample t-test was used to detect enamel erosion differences between negative and positive groups, while a one-way analysis of variance (ANOVA), followed by post hoc Dunnett's test and Tukey's HSD test, were used to detect differences between the sets of treatment groups and negative control groups, or among all experimental groups. All tests utilized a 0.05 level of significance, and statistical analyses were performed using the statistical package SAS ${ }^{\circledR}$ System version 9.3 (SAS Institute Inc., Cary, NC, USA). Flow Chart 1 illustrates the experimental procedures of this study.

\section{RESULTS}

\section{Detecting the Difference in the Amount of Enamel Erosion between Positive and Negative Control Groups}

Based on the two-sample t-test, the data revealed that there was a significant difference in the mean amount of enamel erosion $(p<0.0001)$. The mean enamel erosion for positive control group was significantly greater than that observed in negative control group (mean: 23.50 for positive control vs 2.65 for negative control) (Table 1).

\section{Detecting the Difference in the Amount of Enamel Erosion between a Set of Treatment Groups and Negative Control Group}

Results of one-way ANOVA revealed that there was a significant effect for the type of treatments on the amount of enamel erosion $[\mathrm{F}(9,50)=25.19 ; \mathrm{p}<0.0001]$. The post hoc Dunnett's test indicated that there was no significant difference between the negative control group and each of treatment groups ( $\mathrm{p}>0.05$ for all instances), except for NW_10 treatment group ( $\mathrm{p}<0.0001)$. The data showed that the mean amount of enamel erosion for NW_10 was significantly greater than that observed in negative control group (mean: 14.82 for NW_10 vs 2.65 for negative control). Table 2 provides detailed results from the post hoc Dunnett's test. Moreover, the post hoc Tukey's HSD test indicated that the mean amount
Flow Chart 1: Experimental design of this study

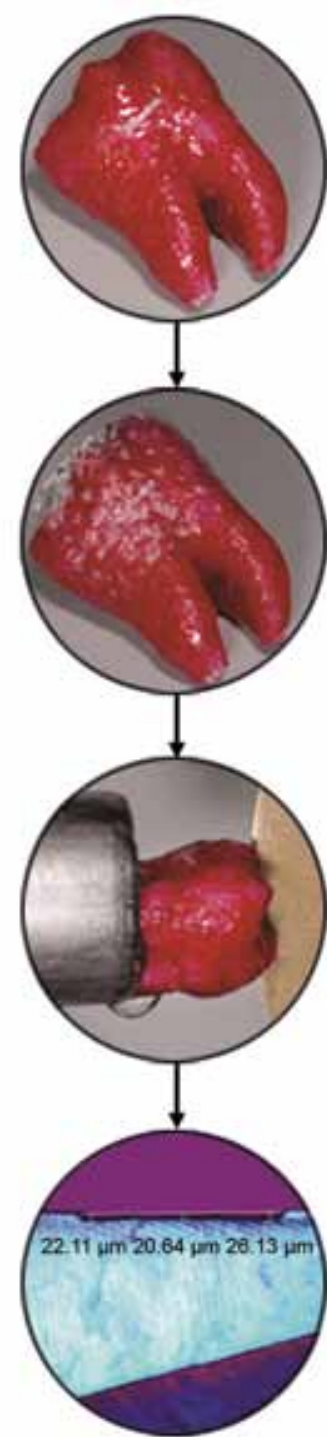

Standardized window painting with nail varnish $(1 \times 4 \mathrm{~mm})$

Tooth whitening by experimental group

Sectioning with microtome (100 um)

\section{Polarized light microscopy}

Table 2: Pairwise comparisons of mean amount of enamel erosion $(\mu \mathrm{m})$ between negative control group and a set of treatment groups

\begin{tabular}{llll}
\hline Experimental group & $N$ & $\begin{array}{l}\text { Mean enamel } \\
\text { erosion (SD) }\end{array}$ & $\begin{array}{l}\text { Pairwise group } \\
\text { comparisons }^{* *}\end{array}$ \\
\hline Negative control & 6 & $2.65(1.11)$ & $\mathrm{A}$ \\
NW_10 & 6 & $23.50(3.91)$ & $\mathrm{B}$ \\
NW_5 & 6 & $3.62(0.95)$ & $\mathrm{A}$ \\
SB_5 & 6 & $3.16(1.48)$ & $\mathrm{A}$ \\
SB_10 & 6 & $3.10(1.50)$ & $\mathrm{A}$ \\
CWS_5 & 6 & $3.10(1.99)$ & $\mathrm{A}$ \\
CWS_1 & 6 & $3.04(1.53)$ & $\mathrm{A}$ \\
SB_1 & 6 & $2.95(1.46)$ & $\mathrm{A}$ \\
CWS_10 & 6 & $2.80(1.45)$ & $\mathrm{A}$ \\
NW_1 & 6 & $2.75(1.11)$ & $\mathrm{A}$ \\
\hline
\end{tabular}

${ }^{* *}$ Means with the same letter are not significantly different using the post hoc Dunnett's test $(p>0.05)$

of enamel erosion for NW_10 was significantly greater than that observed in the other nine experimental groups, while no significant difference was found among those nine groups. Figures $1 \mathrm{~A}$ to $\mathrm{D}$ illustrate the representative images for the measurement of erosion depth. 

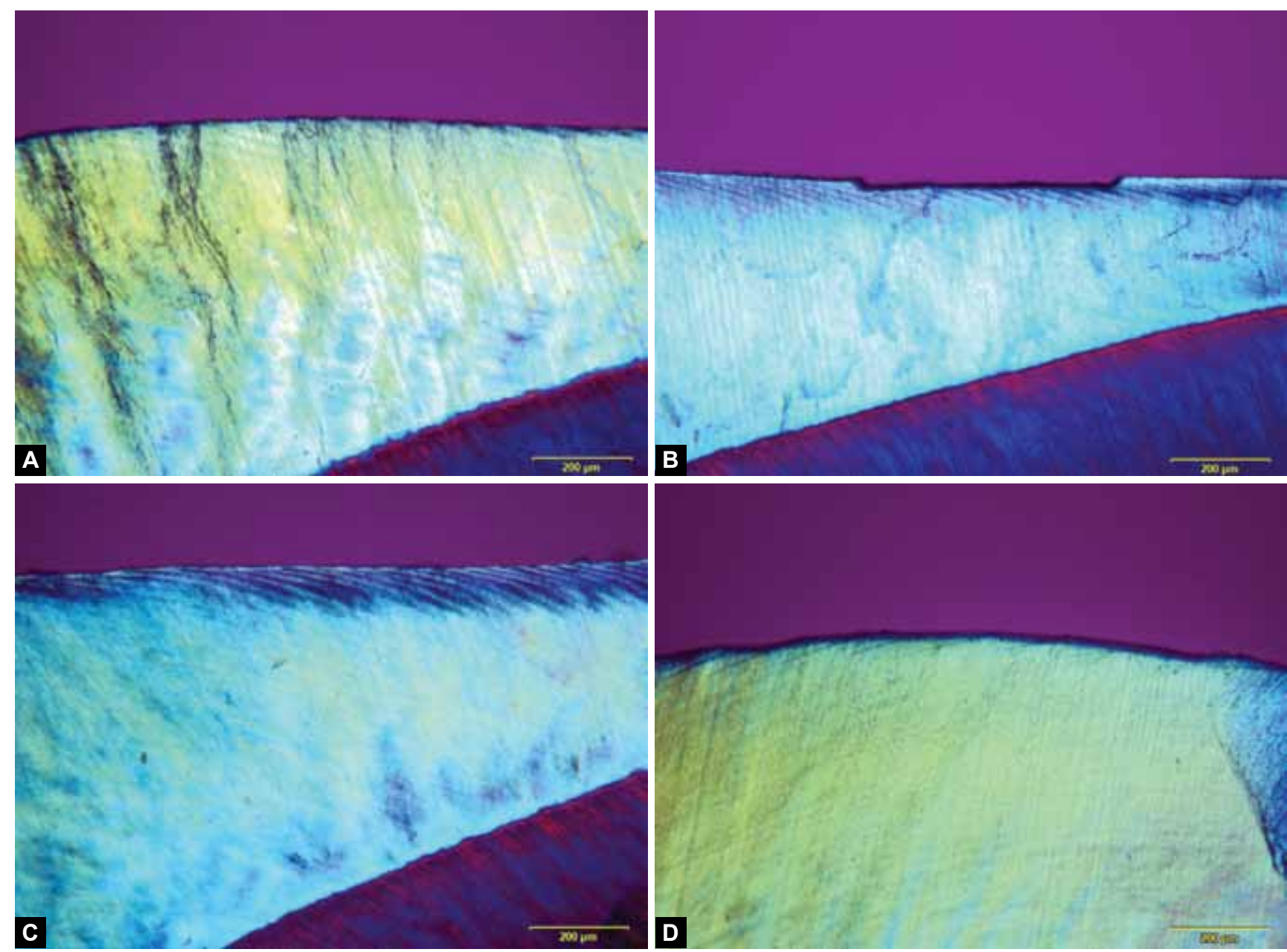

Figs 1A to D: Representative micrographs: (A) Negative control, (B) positive control, (C) crest whitestrips $10 \times$ group and (D) natural white $10 \times$ group

\section{DISCUSSION}

The availability of over-the-counter products and various DIY whitening modalities as advocated over the Internet has provided better access to tooth whitening to the public. Whilez professionally applied in-office whitening and dentist administered home whitening are supervised throughout the process, the use of OTC products and DIY modalities without the supervision of the dentist has raised several potential concerns such as lack of establishing diagnosis of the etiology of the discoloration, abuse due to overuse of whitening material leading to adverse events on the enamel surface, and inability to monitor the progress of tooth color change. ${ }^{1,14-16}$

Bleachorexia has been defined as an obsession of tooth whitening. There is no estimated number on the population affected but the risk of patient abusing whitening agents has increased over time, with adolescents being a particularly at risk group for overuse of whitening agents. ${ }^{12}$ Therefore, the use of tooth whiteners beyond manufacturer's recommendation has become a central issue to be further addressed and warrant additional investigation. The purpose of this study was to exceed the whitening dosages set by manufacturers of various whitening modalities to determine how much excess application of whitening products would lead to critically eroded enamel. The hypothesis to be tested was that use of whitening materials beyond manufacturer's recommendation would not increase erosion amount.

Based on the results our hypothesis was rejected, for certain whitening modalities whitening beyond manufacturer's recommendations increased the erosion amount in the enamel as measured with polarized light microscopy. It is important to point out that the ISO 28399 defines the significant threshold point as three times the amount of erosion seen in an hour of acid attack by citric acid with a $\mathrm{pH}$ of 3.9 as measured with profilometry. ${ }^{13}$ The highest erosion depth in our study was found in the NW_10 group exhibiting a mean of $14.82 \mu \mathrm{m}$. Although this drop was significant when compared to other groups it did not reach the drop observed in the positive control group $(23.50 \mu \mathrm{m})$. An interesting aspect was to observe that the overuse of crest whitening strips and of DIY whitening with strawberries did not increase the erosion depth when compared to the recommended protocol. However, a study that evaluated the effect of various tooth whitening modalities showed that the use 
of strawberry puree caused a significant drop in Knoop hardness. ${ }^{6}$ They attributed this drop to the fact that there may have been small chunks of strawberry present in the mixture that contributed a to a low $\mathrm{pH}$ in localized areas.

It is important to note that this study was an in vitro study on extracted teeth that does not reflect the full dynamics of the oral environment, e.g. the effects of saliva and the positive outward pulpal pressure associated with vital teeth. Nevertheless, the results of our study pave the way for possible use of the polarized light microscope in evaluating erosion potential regarding abuse of whitening materials. Additional studies could evaluate the effect on dentin and other restorative materials to further establish the safety of various tooth whitening modalities to ultimately benefit the general public.

\section{CONCLUSION}

Over-the-counter whitening products have been shown to have little danger of enamel erosion when used according to manufacturer guidelines. However, overuse of these products may lead to a significant amount of erosion. Within the limitations of this study it can be concluded that enamel erosion due to the overuse of whitening products varies for different modalities and products. Therefore, caution is advised when using certain overthe-counter products beyond recommended guidelines, as there is potential for enamel erosion.

\section{ACKNOWLEDGMENT}

This study was supported by The University of Iowa Dental Research Grant and received the 1st Prize of the ADA Pre-Doctoral Poster Session at the Local AADR Meeting in Iowa City, IA, USA.

\section{REFERENCES}

1. American Dental Association Council on Scientific Affairs. Tooth Whitening/Bleaching: Treatment Considerations for Dentists and Their Patients. Chicago: ADA 2009.
2. Kwon SR, Meharry M, Oyoyo U, Li Y. Efficacy of do-it-yourself whitening as compared to conventional tooth whitening modalities: an in vitro study. Oper Dent 2015;40:E21-27.

3. Matis BA, Cochran MA, Eckert G. Review of the effectiveness of various tooth whitening systems. Oper Dent 2009;34: 230-235.

4. Auschill TM, Hellwig E, Schmidale S, Schulean A, Arweiler NB. Efficacy, side-effects and patients' acceptance of different whitening techniques (OTC, in-office, at-home). Oper Dent 2005;30:156-163.

5. Kwon SR, Wang J, Oyoyo U, Li Y. Evaluation of whitening efficacy and erosion potential of four different over-thecounter whitening products. Am J Dent 2013;26:356-360.

6. Kwon SR, Kurti SR, Oyoyo U, Li Y. Effect of various tooth whitening modalities on microhardness, surface roughness and surface morphology of the enamel. Odontol 2015;103: 274-279.

7. Engle K, Hara AT, Matis, B, Eckert GJ, Zero DT. Erosion and abrasion of enamel and dentin associated with at-home whitening: an in vitro study. J Am Dent Assoc 2010;141:546-551.

8. Lewinstein I, Fuhrer N, Churaru N, Cardash H. Effect of different peroxide whitening regimens and subsequent fluoridation on the hardness of human enamel and dentin. J Prosthet Dent 2004;92:337-342.

9. Jiang $\mathrm{T}$, Ma X, Wang $\mathrm{Y}$, Tong $\mathrm{H}$, Shen $\mathrm{X}, \mathrm{Hu} \mathrm{Y}, \mathrm{Hu}$ J. Investigation of the effects of $30 \%$ hydrogen peroxide on human tooth enamel by Raman scattering and laser-induced fluorescence. J Biomed Opt 2008;13:014019.

10. Mei L, Busscher HJ, van der Mei HC, Ren Y. Influence of surface roughness on streptococcal adhesion forces to composite resins. Dent Mater 2011;27:770-778.

11. Joiner A. Review of the effects of peroxide on enamel and dentine properties. J Dent 2007;35:889-896.

12. Lee SS, Zhang W, Lee HD, Li Y. Tooth whitening in children and adolescents: a literature review. Ped Dent 2005;27: 362-368.

13. International Organization for Standardization. DentistryProducts for external tooth whitening. International Standard 28399 2011;1(1):6-10.

14. Cubbon T, Ore D. Hard tissue and home tooth whiteners. CDS Rev 1991;84:32-35.

15. Hammel S. Do-it-yourself tooth whitening is risky. US News and World Report 1998;66.

16. Li Y. Safety controversies in tooth bleaching. Dent Clin North Am 2011;55:229-239. 